\title{
Successful Management of Immune-Mediated Hemolytic Anemia Secondary to Infection with Cytauxzoon felis and Feline Immunodeficiency Virus
}

\author{
Hyeong-Il Choi*, Joonyong Kim*, Jae-Ik Han ${ }^{* *}$ and Ha-Jung Kim*1 \\ * Department of Internal Medicine, College of Veterinary Medicine, Chonnam National University, Gwangju 61186, Korea \\ **Laboratory of Wildlife Medicine, College of Veterinary Medicine, Chonbuk National University, Iksan 54596, Korea
}

(Received: June 04, 2020 / Revised: August 11, 2020 / Accepted: August 11, 2020)

\begin{abstract}
Cytauxzoonosis is caused by Cytauxzoon felis (C. felis) in wild and domestic cats. However, cytauxzoonosis is uncommon in Asia. Additionally, clinical reports of $C$. felis infection along with associated complications are rare. A seven-year-old neutered male Maine Coon cat was presented with acute dyspnea and lethargy despite the absence of a history of overseas travel. Mild regenerative anemia and autoagglutination were detected in hematological investigations. The parasitic and viral PCR assays revealed infection with $C$. felis and feline immunodeficiency virus (FIV). Thoracic radiographs showed pleural effusion with secondary bacterial infection. Ultimately, a diagnosis of infection-induced secondary immune-mediated hemolytic anemia (IMHA) and pyothorax was established. The cat was treated with a combination of atovaquone, prednisolone, and cyclosporine over 6 months and the final treatment was completed 8 months after initiation of therapy. This is the first report of its kind demonstrating successful management of feline IMHA and fatal pyothorax induced by FIV and C. felis in South Korea.
\end{abstract}

Key words : anemia, atovaquone, Cytauxzoon felis, FIV, IMHA.

\section{Introduction}

Cytauxzoonosis is an emerging tick-borne feline disease caused by Cytauxzoon felis affecting wild and domestic felids (9). For many years, cytauxzoonosis in domestic cats was reported only in North and South America (8) and the species in European cases differ from $C$. felis, which causes infection and disease in the United States (13). Dermacentor variabilis and Amblyomma americanum have been shown to be the tick vectors for $C$. felis (21). Recent studies have shown that domestic cats can harbor subclinical infections and may act as reservoirs $(8,12)$. In domestic cats, natural and experimental infections have led to a rapid course of illness and death, typically in fewer than 5 days $(9,21)$. Information regarding the epidemiological distribution, clinical presentation, genetics, and pathogenicity of infection by $C$. felis in Asia is scant. The cat in the present case had no history of traveling abroad to known epidemic areas. We report the first C. felis infection case in South Korea.

\section{Case Presentation}

A 7-year-old neutered male Maine Coon cat was presented with a history of acute onset of dyspnea, lethargy, and anorexia. The cat was born and raised in South Korea and had never travelled abroad. No health issues had previously been diagnosed, and its past history was unremarkable. At physical examination, the patient was tachypneic and dys-

Corresponding author.

E-mail : kimhj614@jnu.ac.kr pneic with a mildly pale mucous membrane. Capillary refill time was approximately $2 \mathrm{sec}$. Moderate hypochromic anemia with mild neutrophilic leukocytosis was noted (Table 1). Abnormality of serum biochemistry was detected only decreased levels of albumin (2.1 g/dL; reference range: 2.2-4.0).

Thoracic radiographs verified the cause of dyspnea to be pleural effusion (Fig 1), which was sampled by therapeutic thoracocentesis for cytology and bacterial culture. The fluid was revealed to be an exudate containing degenerative neutrophils with phagocytized bacteria and sensitivity test was submitted (Fig 2).

The cat was hospitalized and oxygen therapy was started. Additionally, intravenous (IV) crystalloid fluid therapy was administered to correct the dehydration and provide fluid therapy maintenance. Antibiotic treatment with cefotaxime $(50 \mathrm{mg} / \mathrm{kg}, \mathrm{IV}$, b.i.d.) and metronidazole (15 mg/kg, IV, s.i.d.) was started, and furosemide (4 mg/kg, IV, b.i.d.) and dexamethasone $(0.5 \mathrm{mg} / \mathrm{cat}, \mathrm{IV}$, s.i.d.) were administered to treat the pleural effusion. After intensive care, the clinical signs improved, and then cardiac problems were ruled out by echocardiography.

The patient was discharged and $\mathrm{CBC}$ on day 10 showed more severe anemia (HCT: 20.6\%; reference range: 30.352.3). A blood smear test for anemia revealed small inclusions within erythrocytes (Fig 3). The inclusions were annular, suggesting the possibility of small piroplasmid parasite infection. Marked agglutination of erythrocytes was also noted with saline dilution on microscope. A real-time polymerase chain reaction (PCR) panel (Anemia RealPCR ${ }^{\mathrm{TM}}$ Panel; IDEXX Reference Laboratories, Westbrook, ME, USA) confirmed C. felis and FIV (subtype-b) infection. Ultimately, $C$. felis and FIV infection-induced secondary IMHA 
Table 1. Summary of blood cell counts follow-ups from the presentation

\begin{tabular}{lllllll}
\hline \hline Parameter (unit) & Day 0 & 2 weeks & 1 month & 2 months & 8 months & Reference range \\
\hline RBC $(\mathrm{M} / \mu \mathrm{L})$ & 4.76 & 6.92 & 7.34 & 7.39 & 8.15 & $6.54-12.2$ \\
Hematocrit $(\%)$ & 26.5 & 34.8 & 38.9 & 35.2 & 39.1 & $30.3-52.3$ \\
RETIC $(\mathrm{K} / \mu \mathrm{L})$ & 120.4 & 9.7 & 29.3 & 14.0 & 11.6 & $3-50$ \\
Hemoglobin $(\mathrm{g} / \mathrm{dL})$ & 8.1 & 11.5 & 12.8 & 11.6 & 13.2 & $9.8-16.2$ \\
MCV $(\mathrm{fL})$ & 55.7 & 50.3 & 53.0 & 47.6 & 48.0 & $35.9-53.1$ \\
MCHC $(\mathrm{g} / \mathrm{dL})$ & 30.6 & 33.0 & 32.9 & 33.0 & 33.8 & $28.1-35.8$ \\
WBC $(\mathrm{K} / \mu \mathrm{L})$ & 51.12 & 10.69 & 11.06 & 13.67 & 11.46 & $2.87-17.02$ \\
Neutrophils $(\mathrm{K} / \mu \mathrm{L})$ & 3.38 & 9.95 & 5.87 & 10.80 & 5.01 & $1.48-10.29$ \\
Eosinophils $(\mathrm{K} / \mu \mathrm{L})$ & 0.50 & 0.11 & 1.80 & 0.87 & 1.39 & $0.17-1.57$ \\
Monocytes $(\mathrm{K} / \mu \mathrm{L})$ & 13.23 & 0.21 & 0.48 & 0.43 & 0.55 & $0.05-0.67$ \\
Lymphocytes $(\mathrm{K} / \mu \mathrm{L})$ & 33.86 & 0.42 & 2.83 & 1.55 & 4.47 & $0.92-6.88$ \\
Platelets $(\mathrm{K} / \mu \mathrm{L})$ & 244 & 471 & 319 & 341 & 277 & $151-600$ \\
\hline
\end{tabular}

RBC: red blood cells count; RETIC: reticulocytes; MCV: mean corpuscular volume; MCHC: mean corpuscular hemoglobin concentration; WBC: white blood cells count.
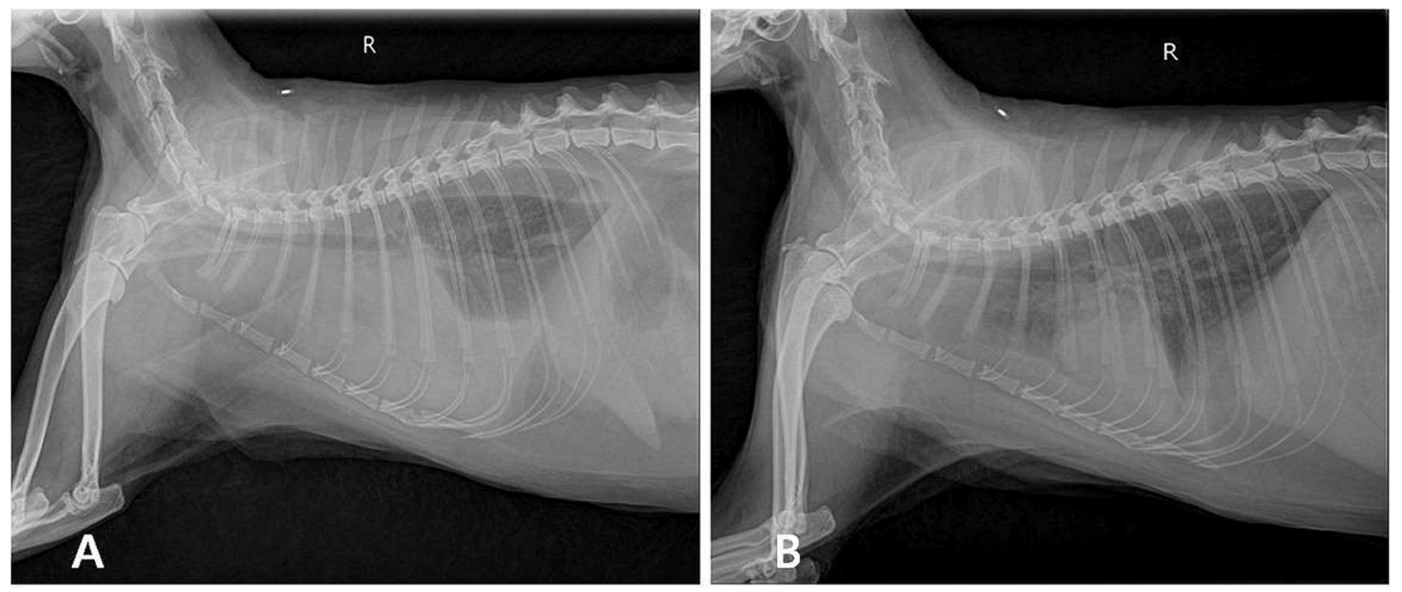

Fig 1. (A) Right lateral thoracic radiograph of a cat with fluid opacity in the pleural space. (B) Decreased pleural effusion and pulmonary edema $36 \mathrm{~h}$ after treatment.

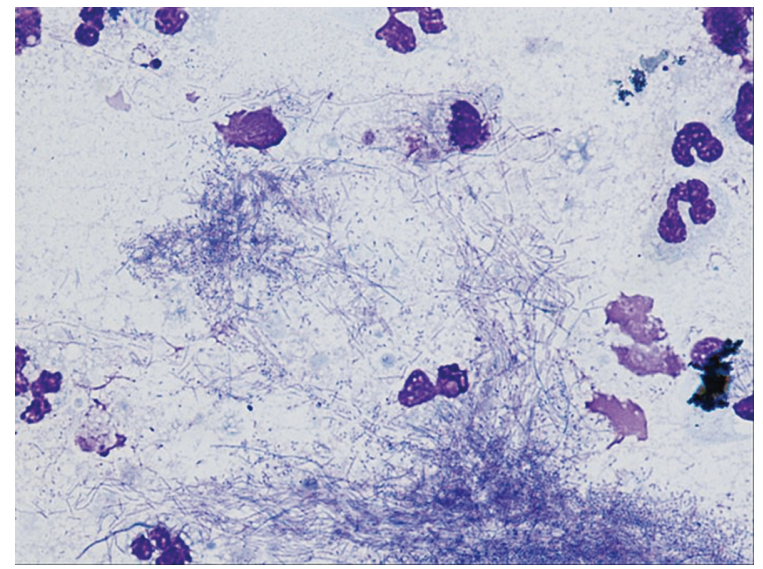

Fig 2. Hematoxylin and eosin (H\&E) stain of the pleural fluid, revealing large amounts of bacteria and degenerated neutrophils and macrophages $(\times 1,000$ magnitude $)$.

and pyothorax were diagnosed.

Treatment included oral atovaquone $(15 \mathrm{mg} / \mathrm{kg}$, p.o., t.i.d., Glaxo Wellcome, London, UK), azithromycin $(10 \mathrm{mg} / \mathrm{kg}$, p.o., s.i.d., Pfizer, NY, USA), prednisolone ( $2 \mathrm{mg} / \mathrm{kg}$, p.o.,

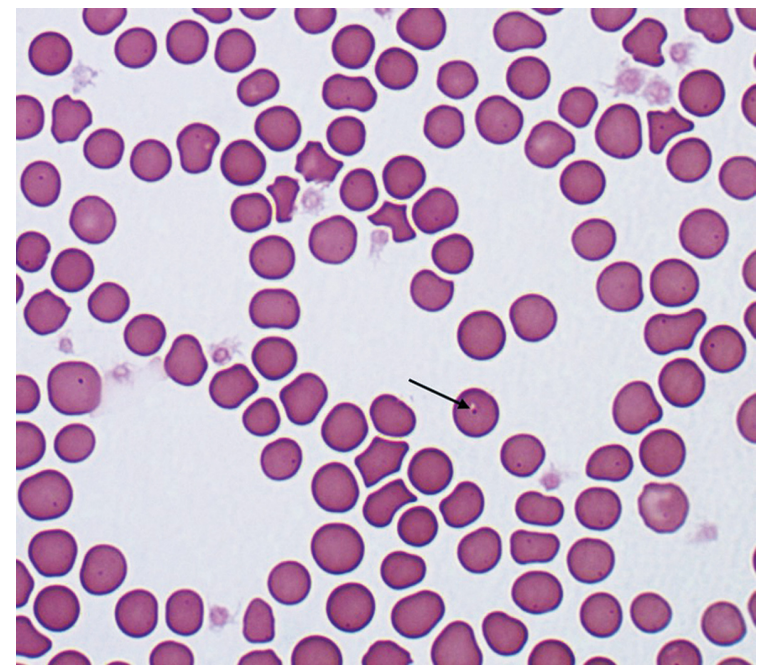

Fig 3. Blood smear with H\&E staining showing Cytauxzoon felis piroplasms (arrow) in erythrocytes $(\times 1,000)$.

b.i.d., Lloyd Inc, Shenandoah, USA), and cyclosporine (5 $\mathrm{mg} / \mathrm{kg}$, p.o., b.i.d., Chong Kun Dang, Seoul, South Korea) for $C$. felis infection-induced IMHA and doxycycline $(5 \mathrm{mg}$ / 
kg, p.o., b.i.d., Parsippany-Troy Hills, NJ, USA) and metronidazole $(10 \mathrm{mg} / \mathrm{kg}$, p.o., b.i.d., Pfizer, NY, USA) for pyothorax. Two weeks after treatment, the patient exhibited excellent health, appetite was good, and parasitemia was absent according to a blood smear microscopic examination. Anemia was resolved (HCT: 34.8\%; reference range: 30.3-52.3), and realtime PCR test yielded negative results. However, 3 weeks after treatment, the cat was presented with a new episode of vomiting twice a week. A feline pancreatic lipase immunoreactivity assay (SNAP fPLI kit; IDEXX Reference Laboratories) yielded abnormal results. Supportive therapy consisted of intravenous fluids, antiemetic drugs, and fresh frozen plasma to treat pancreatitis, which recovered rapidly without other clinical signs. Abnormalities revealed by blood work gradually resolved (Table 1). Blood work, including slide smear tests and complete blood count (CBC), was regularly checked ( 2 weeks, 1 month, 2 months, and 8 months later). No definitive organisms were observed on the smears and no protozoans were detected using real-time PCR test (IDEXX Reference Laboratories).

Clinical presentation, blood tests, morphological and molecular investigation confirm the presence of $C$. felis in cats. More specific and sensitive methods, such as PCR, can also be adopted for confirmation (4). In the present case, hemolytic anemia with intraerythrocytic inclusions and real-time PCR results indicated $C$. felis infection. The organism is an arthropod-borne organism, which may be one of the causes of secondary IMHA $(8,9)$. Secondary IMHA in cats is relatively common compared to dogs and can be caused by infections (e.g., blood parasites and viruses), inflammations, drugs, and neoplasia $(8,10,16,19)$. The present case was in emergency, because pyothorax occurred with dyspnea and severe respiratory distress in the cat. Pyothorax may be caused by immunosuppressive conditions. The cat needed aggressive antibiotic therapy based on a sensitivity test with the removal of pleural effusion. Secondary IMHA caused by immunosuppressive agents and anemia caused by infectious parasites were also treated at the same time. The urgent and emergency status was managed effectively and the patient became stable.

\section{Discussion}

Cytauxzoon sp. infection has been reported to be significantly related to FIV infection $(7,15)$, which frequently causes immune suppression in infected cats. Clinical signs of FIV infection can arise from direct viral effects or other infections secondary to the development of immunodeficiency $(15,17)$. In this case, immune suppression caused by FIV may be a factor for susceptibility to $C$. felis and bacterial pleural infection, or FIV could directly cause IMHA through simultaneous infection with $C$. felis. Therefore, both infectious agents could exert effects on systemic immunity. Chronic infections or infections caused by opportunistic pathogens such as FIV can trigger monocytosis and lymphocytosis in some cats (14). In this case, blood work revealed monocytosis and lymphocytosis. However, additional diagnostic approaches may identify treatable etiologies because, in many cases, the clinical signs associated with FIV can be caused by opportu- nistic infections.

Cytauxzoonosis caused by $C$. felis has been considered a fatal disease, with mortality approaching $90 \%$ among infected cats (3). Treatment for $C$. felis can be problematic because the infection is not typically resolved by standard cytauxzoonosis therapies. The cat in this case was treated with atovaquone and azithromycin, and the response was favorable. A recent study demonstrated that therapy combining atovaquone and azithromycin is a safe and effective treatment for cytauxzoonosis in cats (2). Thirty-two of 53 domestic cats $(60 \%)$ treated with atovaquone and azithromycin survived to discharge, whereas only $26 \%$ of domestic cats ( 7 of 27 cats) treated with imidocarb survived (5). Atovaquone targets $C$. felis cytochrome $b$, and azithromycin targets the mitochondrial ribosomes of the parasite (11). However, atovaquone remains an expensive drug, and few reports have described its side effects (6). In this case, 3 weeks after therapy with atovaquone, the cat was diagnosed with acute pancreatitis. In human medicine, atovaquone has been reported to induce pancreatitis (1). Another recent study also reported a potential association between pancreatitis and IMHA in cats (22).

\section{Conclusion}

In conclusion, this case report is the first to describe $C$. felis infection with FIV and the subsequent occurrence of secondary IMHA in a domestic cat in South Korea. The fatal systemic complications caused by infectious agents were successfully managed with anti-parasitic drugs, antibiotics, and immunosuppressive agents. The transmission epidemiology of $C$. felis infection in South Korea remains unclear and additional studies are needed.

\section{Acknowledgement}

This research was supported by Basic Science Research Program through the National Research Foundation of Korea (NRF), which is funded by the Ministry of Education (NRF2016R1D1A3B04934798).

\section{Conflict of Interest}

The authors declared no potential conflicts of interest with respect to the research, authorship, and/or publication of this article.

\section{References}

1. Birkenheuer AJ, Marr HS, Warren C, Acton AE, Mucker EM, Humphreys JG, Tucker MD. Cytauxzoon felis infections are present in bobcats (Lynx rufus) in a region where cytauxzoonosis is not recognized in domestic cats. Vet Parasitol 2008; 153: 126-130.

2. Cohn LA, Birkenheuer AJ, Brunker JD, Ratcliff ER, Craig AW. Efficacy of atovaquone and azithromycin or imidocarb dipropionate in cats with acute cytauxzoonosis. J Vet Intern Med 2011; 25: 55-60.

3. Frontera-Acevedo K, Balsone NM, Dugan MA, Makemson CR, Sellers LB, Brown HM, Peterson DS, Creevy KE, Garner $\mathrm{BC}$, Sakamoto K. Systemic immune responses in Cytauxzoon 
felis-infected domestic cats. Am J Vet Res 2013; 74: 901-909.

4. Hegarty BC, Qurollo BA, Thomas B, Park K, Chandrashekar R, Beall MJ, Thatcher B, Breitschwerdt EB. Serological and molecular analysis of feline vector-borne anaplasmosis and ehrlichiosis using species-specific peptides and PCR. Parasit Vectors 2015; 8: 320.

5. Holman PJ, Snowden KF. Canine hepatozoonosis and babesiosis, and feline cytauxzoonosis. Vet Clin North Am Small Anim Pract 2009; 39: 1035-1053.

6. Karch FE, Lasagna L. Adverse drug reactions. A critical review. JAMA 1975; 234: 1236-1241.

7. Kohn B, Weingart C, Eckmann V, Ottenjann M, Leibold W. Primary immune-mediated hemolytic anemia in 19 cats: diagnosis, therapy, and outcome (1998-2004). J Vet Intern Med 2006; 20: 159-166.

8. Little SE, Barrett AW, Nagamori Y, Herrin BH, Normile D, Heaney K, Armstrong R. Ticks from cats in the United States: Patterns of infestation and infection with pathogens. Vet Parasitol 2018; 257: 15-20.

9. Lloret A, Addie DD, Boucraut-Baralon C, Egberink H, Frymus T, Gruffydd-Jones T, Hartmann K, Horzinek MC, Hosie MJ, Lutz H, Marsilio F, Pennisi MG, Radford A D, Thiry E, Truyen U, Möstl K. Cytauxzoonosis in cats: ABCD guidelines on prevention and management. J Feline Med Surg 2015; 17: 637-641.

10. Merten N, Weingart C, Kohn B. Causes, diagnostics and course of disease in 194 cats with anemia. Berl Munch Tierarztl Wochenschr 2015; 128: 384-393.

11. Motzel SL, Wagner JE. Treatment of experimentally induced cytauxzoonosis in cats with parvaquone and buparvaquone. Vet Parasitol 1990; 35: 131-138.

12. Nagamori Y, Slovak JE, Reichard MV. Prevalence of Cytauxzoon felis infection in healthy free-roaming cats in north-central Oklahoma and central Iowa. JFMS Open Rep 2016; 2: 2055116916655174
13. Nentwig A, Meli ML, Schrack J, Reichler IM, Riond B, Gloor C, Howard J, Hofmann-Lehmann R, Willi B. First report of Cytauxzoon sp. infection in domestic cats in Switzerland: natural and transfusion-transmitted infections. Parasit Vectors 2018; 11: 292.

14. Ottenjann M, Weingart C, Arndt G, Kohn B. Characterization of the anemia of inflammatory disease in cats with abscesses, pyothorax, or fat necrosis. J Vet Intern Med 2006; 20: 11431150.

15. Penzhorn BL, Schoeman T, Jacobson LS. Feline Babesiosis in South Africa: a review. Ann N Y Acad Sci 2004; 1026: 183186.

16. Swann JW, Szladovits B, Glanemann B. Demographic Characteristics, survival and prognostic factors for mortality in cats with primary immune-mediated hemolytic anemia. J Vet Intern Med 2016; 30: 147-156.

17. Switzer JW, Jain NC. Autoimmune hemolytic anemia in dogs and cats. Vet Clin North Am Small Anim Pract 1981; 11: 405-420.

18. Wardrop KJ. Coombs' testing and its diagnostic significance in dogs and cats. Vet Clin North Am Small Anim Pract 2012; 42: 43-51.

19. White C, Reine N. Feline nonregenerative anemia: pathophysiology and etiologies. Compend Contin Educ Vet 2009; 31: E1-7.

20. Zieman EA, Jiménez FA, Nielsen CK. Concurrent examination of bobcats and ticks reveals high prevalence of Cytauxzoon felis in southern illinois. J Parasitol 2017; 103: 343-348.

21. Zieman EA, Nielsen CK, Jiménez FA. Chronic Cytauxzoon felis infections in wild-caught bobcats (Lynx rufus). Vet Parasitol 2018; 252: 67-69.

22. Zoia A, Drigo M. Association between pancreatitis and immune-mediated haemolytic anaemia in cats: A crosssectional study. J Comp Pathol 2017; 156: 384-388. 